\title{
Edge regular properties of truncations of intuitionistic fuzzy graphs
}

\author{
A. Nagoor Gani and H. Sheik Mujibur Rahman \\ PG \& Research Department of Mathematics, Jamal Mohamed College (Autonomous) \\ Tiruchirappalli - 620020, India \\ e-mails: ganijmc@yahoo.co.in, mujeebmaths@gmail.com
}

Received: 5 October $2017 \quad$ Revised: 30 November $2017 \quad$ Accepted: 2 December 2017

\begin{abstract}
In this paper, the adjacency sequence of intuitionistic fuzzy graph is defined. Also the degree of an edge in truncations of intuitionistic fuzzy graphs are obtained and edge regular properties of truncations of intuitionistic fuzzy graphs are discussed.
\end{abstract}

Keywords: Adjacency sequence, Edge regular intuitionistic fuzzy graph, Truncation of intuitionistic fuzzy graph, Upper and lower truncations of intuitionistic fuzzy graph.

2010 Mathematics Subject Classification: 03E72, 03F55.

\section{Introduction}

Intuitionistic Fuzzy Graph theory was introduced by A. Shannon and K. Atanassov. In [8] R. Parvathi and M. G. Karunambigai introduced intuitionistic fuzzy graph as a special case of Atanassov's IFG. In [3] M. G. Karunambigai and Palanivel introduce the edge regular intuitionistic fuzzy graph. In [9] K. Radha and N. Kumaravel introduced the edge degree and the edge regular properties of truncations of fuzzy graphs. In [6] Akram and Alshehri introduces the intuitionistic fuzzy cycle intuitionistic fuzzy trees. A. Nagoor Gani and H. Sheik Mujibur Rahman introduced the lower and upper truncations of intuitionistic fuzzy graphs in [7]. In this paper we introduce the degree of an edge in truncations of intuitionistic fuzzy graphs and edge regular properties of truncations of intuitionistic fuzzy graphs.

\section{Preliminaries}

Let $G:(V, E)$ be an intuitionistic fuzzy graph. Then the degree of a vertex $v$ is defined by $(v)=$ $\left(d_{\mu}(v), d_{v}(v)\right)$, where $d_{\mu}(v)=\sum_{u \neq v} \mu_{2}(v, u)$ and $d_{v}(v)=\sum_{u \neq v} v_{2}(v, u)$. 
Let $G:(V, E)$ be an IFG and let $e_{i j} \in E$ be an edge in $G$. Then the degree of an edge $e_{i j} \in$ $E$ is defined as $d_{\mu}\left(e_{i j}\right)=d_{\mu}\left(v_{i}\right)+d_{\mu}\left(v_{j}\right)-2 \mu_{2}\left(v_{i}, v_{j}\right)$ (or)

$d_{\mu}\left(e_{i j}\right)=\sum_{v_{i} v_{k} \in E, k \neq j} \mu_{2}\left(v_{i}, v_{k}\right)+\sum_{v_{k} v_{j} \in E, k \neq i} \mu_{2}\left(v_{k}, v_{j}\right)$

$d_{v}\left(e_{i j}\right)=d_{v}\left(v_{j}\right)+d_{v}\left(v_{j}\right)-2 v_{2}\left(v_{i}, v_{j}\right)$ (or)

$d_{v}\left(e_{i j}\right)=\sum_{v_{i} v_{k} \in E, k \neq j} v_{2}\left(v_{i}, v_{k}\right)+\sum_{v_{k} v_{j} \in E, k \neq i} v_{2}\left(v_{k}, v_{j}\right)$, and the edge degree of $G$ is defined by $d_{G}\left(e_{i j}\right)=\left(d_{\mu}\left(e_{i j}\right), d_{v}\left(e_{i j}\right)\right)$. Let $G=\langle V, E\rangle$ be an intuitionistic fuzzy graph. If each edge in $G$ has the same degree $\left(l_{1}, l_{2}\right)$, then $G$ is said to be an edge regular intuitionistic fuzzy graph.

Let $\left(\mu_{1}, v_{1}\right)$ be an intuitionistic fuzzy subset of a set $V$. The lower and upper truncations of the intuitionistic fuzzy set $\left(\mu_{1}, v_{1}\right)$ at level $t, 0<t \leq 1$, are the intuitionistic fuzzy subsets $\left(\mu_{1}, v_{1}\right)_{(t)}$ and $\left(\mu_{1}, v_{1}\right)^{(t)}$ defined respectively by

$$
\begin{aligned}
& \left(\mu_{1}, v_{1}\right)_{(t)}(u)=\left\{\begin{array}{c}
\left(\mu_{1}, v_{1}\right)(u), \text { if } u \in \mu_{1}^{t} \text { and } u \in v_{1}{ }^{t} \\
0, \text { if } u \notin \mu_{1}^{t}(\text { or }) u \notin v_{1}{ }^{t}
\end{array}\right. \\
& \left(\mu_{1}, v_{1}\right)^{(t)}(u)=\left\{\begin{array}{c}
t, \text { if } u \in \mu_{1}^{t} \text { and } u \in v_{1}^{t} \\
\left(\mu_{1}, v_{1}\right)(u), \text { if } u \notin \mu_{1}^{t}(\text { or }) u \notin v_{1}{ }^{t}
\end{array}\right.
\end{aligned}
$$

where $\mu_{1}^{t}=\left\{u \in V / \mu_{1}(u) \geq t\right\}$ and $v_{1}{ }^{t}=\left\{u \in V / v_{1}(u) \leq t\right\}$. Take $V_{(t)}=\left(\mu_{1}, v_{1}\right)^{t}, \mathrm{E}_{(t)}=$ $\left(\mu_{2}, v_{2}\right)^{t} . \mathrm{G}_{(t)}:\left(\mu_{(t)}, v_{(t)}\right)$ is an intuitionistic fuzzy graph with underlying crisp graph $G_{(t)}^{*}:\left(\mathrm{V}_{(t)}, \mathrm{E}_{(t)}\right)$. This is called the lower truncation of the intuitionistic fuzzy graph $G$ at level $t$. Here $\mathrm{V}_{(t)}$ and $\mathrm{E}_{(t)}$ may be proper subsets of $V$ and $E$, respectively. Now take $V^{(t)}=V, E^{(t)}=E$, then $G^{(t)}:\left(\mu^{(t)}, v^{(t)}\right)$ is an intuitionistic fuzzy graph with underlying crisp graph $G^{(t) *}:\left(V^{(t)}, \mathrm{E}^{(t)}\right)$. This is called the upper truncation of the intuitionistic fuzzy graph $G$ at level $t$.

\section{Degree of an edge in truncations of intuitionistic fuzzy graph}

\subsection{Degree of an edge in lower truncation of intuitionistic fuzzy graph}

$$
\begin{aligned}
& d_{G_{(t)}}(u v)=\left(d_{\mu(G)(t)}(u v), d_{v(G)(t)}(u v)\right) \\
& d_{\mu(G)(t)}(u v)=\sum_{u w \in E_{(t)}, w \neq v} \mu_{2_{(t)}}(u w)+\sum_{w v \in E_{(t)}, w \neq u} \mu_{2_{(t)}}(w v), \forall u v \in E_{(t)} \\
& =\sum_{u w \in E, w \neq v} \mu_{2}(u w)-\sum_{\substack{u w \in E, w \neq v \\
\mu_{2}(u w)<t}} \mu_{2}(u w)+\sum_{w v \in E, w \neq u} \mu_{2}(w v)-\sum_{\substack{w v \in E, w \neq u \\
\mu_{2}(v w)<t}} \mu_{2}(w v), \forall u v \in E_{(t)} \\
& =d_{\mu(G)}(u v)-\sum_{u w \in E, w \neq v} \mu_{2}(u w)-\sum_{\mu_{2}(u w)<t} \mu_{2}(w v), \forall u v \in E_{(t)} \\
& d_{v(G)(t)}(u v)=\sum_{u w \in E(t), w \neq v} v_{2_{(t)}}(u w)+\sum_{\substack{w v \in v_{2}(v w)<t \\
w}} v_{2_{(t)}}(w v), \forall u v \in E_{(t)} \\
& \sum_{u w \in E, w \neq v} v_{2}(u w)-\sum_{\substack{u w \in E, w \neq v \\
v_{2}(u w)>t}} v_{2}(u w)+\sum_{w v \in E, w \neq u} v_{2}(w v)-\sum_{\substack{w v \in E, w \neq u \\
v_{2}(v w)>t}} v_{2}(w v), \forall u v \in E_{(t)}
\end{aligned}
$$


$=d_{v(G)}(u v)-\sum_{\substack{u w \in E, w \neq v \\ v_{2}(u w)>t}} v_{2}(u w)-\sum_{\substack{w v \in E, w \neq u \\ v_{2}(v w)>t}} v_{2}(w v), \forall u v \in E_{(t)}$

\subsection{Degree of an edge in upper truncation of intuitionistic fuzzy graph}

$$
\begin{aligned}
& d_{G}{ }^{(t)}(u v)=\left(d_{\mu(G)}{ }^{(t)}(u v), d_{v(G)}{ }^{(t)}(u v)\right) \\
& d_{\mu(G)}{ }^{(t)}(u v)=\sum_{u w \in E^{(t)}, w \neq v} \mu_{2}^{(t)}(u w)+\sum_{w v \in E^{(t)}, w \neq u} \mu_{2}^{(t)}(w v), \forall u v \in E^{(t)} \\
& =\sum_{u w \in E, w \neq v}^{u w \in E^{(t)}, w \neq v} \mu_{2}(u w)-\sum_{\substack{u w \in E, w \neq v \\
\mu_{2}(u w) \geq t}}^{w v \in E^{(t)}, w \neq u}\left(\mu_{2}(u w)-t\right)+\sum_{w v \in E, w \neq u} \mu_{2}(w v) \\
& -\sum_{\substack{w v \in E, w \neq u \\
\mu_{2}(v w) \geq t}}\left(\mu_{2}(w v)-t\right), \forall u v \in E^{(t)} \\
& =d_{\mu(G)}(u v)-\sum_{\substack{u w \in E, w \neq v \\
\mu_{2}(u w)>t}}\left(\mu_{2}(u w)-t\right)-\sum_{\substack{w v \in E, w \neq u \\
\mu_{2}(v w)>t}}\left(\mu_{2}(w v)-t\right), \forall u v \in E^{(t)} \\
& d_{v(G)}{ }^{(t)}(u v)=\sum_{u w \in E^{(t)}, w \neq v} v_{2}^{(t)}(u w)+\sum_{w v \in E^{(t)}, w \neq u} v_{2}^{(t)}(w v), \forall u v \in E^{(t)} \\
& =\sum_{u w \in E, w \neq v} v_{2}(u w)-\sum_{\substack{u w \in E, w \neq v \\
v_{2}(u w) \leq t}}\left(v_{2}(u w)-t\right)+\sum_{w v \in E, w \neq u} v_{2}(w v) \\
& -\sum_{\substack{w v \in E, w \neq u \\
v_{2}(v w) \leq t}}\left(v_{2}(w v)-t\right), \forall u v \in E^{(t)} \\
& =d_{v(G)}(u v)-\sum_{\substack{u w \in E, w \neq v \\
v_{2}(u w)<t}}\left(v_{2}(u w)-t\right)+\sum_{\substack{w v \in E, w \neq u \\
v_{2}(v w)<t}}\left(v_{2}(w v)-t\right), \forall u v \in E^{(t)}
\end{aligned}
$$

Theorem 3.3: Let $G:(V, E)$ be an intuitionistic fuzzy graph such that $\mu_{2}(u v) \geq t$ and $v_{2}(u v) \leq$ $t, \forall u v \in E$, where $0<t \leq 1$. Then for any $u v \in E_{(t)}, d_{G_{(t)}}(u v)=d_{G}(u v)$

Proof: From (1), for any $u v \in E_{(t)}$

$$
\begin{gathered}
d_{\mu(G)_{(t)}}(u v)=d_{\mu(G)}(u v)-\sum_{\substack{u w \in E, w \neq v \\
\mu_{2}(u w)<t}} \mu_{2}(u w)-\sum_{\substack{w v \in E, w \neq u \\
\mu_{2}(v w)<t \\
\Rightarrow}} \mu_{2}(w v) \\
d_{\mu(G)_{(t)}}(u v)=d_{\mu(G)}(u v) \text {. From }(2), \text { for any } u v \in E_{(t)} \\
d_{v(G)_{(t)}}(u v)=d_{v(G)}(u v)-\sum_{\substack{u w \in E, w \neq v \\
v_{2}(u w)>t}} v_{2}(u w)-\sum_{\substack{w v \in E, w \neq u \\
v_{2}(v w)>t}} v_{2}(w v) \\
\Rightarrow d_{v(G)_{(t)}}(u v)=d_{v(G)}(u v) \\
d_{G_{(t)}}(u v)=\left(d_{\mu(G)_{(t)}}(u v)_{1}, d_{v(G)_{(t)}}(u v)\right)=\left(d_{\mu(G)}(u v), d_{v(G)}(u v)\right)=d_{G}(u v) .
\end{gathered}
$$


Theorem 3.4: Let $G:(V, E)$ be an intuitionistic fuzzy graph such that $\mu_{2}(u v)=c_{1}$ and $v_{2}(u v)=c_{2}, \forall u v \in E$, where $c_{1}$ and $c_{2}$ are constants. Then for any $u v \in E^{(t)}$,

$$
d_{G}^{(t)}(u v)=\left\{\begin{array}{c}
d_{G}(u v), \text { if } c_{1}<t \text { and } c_{2}>t \\
d_{G}(u v)-(c-t) d_{G^{*}}(u v), \text { if } c_{1} \geq t \text { and } c_{2} \leq t
\end{array}\right.
$$

Proof: Let $c_{1}<t$,

$$
\begin{aligned}
& d_{\mu(G)}{ }^{(t)}(u v)=d_{\mu(G)}(u v)-\sum_{\substack{u w \in E, w \neq v \\
\mu_{2}(u w)>t}}\left(\mu_{2}(u w)-t\right)-\sum_{\substack{w v \in E, w \neq u \\
\mu_{2}(v w)>t}}\left(\mu_{2}(w v)-t\right) \\
& =d_{\mu(G)}(u v)-\sum_{\substack{u w \in E, w \neq v \\
c_{1}>t}}\left(c_{1}-t\right)-\sum_{\substack{w v \in E, w \neq u \\
c_{1}>t}}\left(c_{1}-t\right) \\
& \Rightarrow d_{\mu(G)}{ }^{(t)}(u v)=d_{\mu(G)}(u v) \text {. Let } c_{2}>t, \\
& d_{v(G)}{ }^{(t)}(u v)=d_{v(G)}(u v)-\sum_{\substack{u w \in E, w \neq v \\
v_{2}(u w)<t}}\left(v_{2}(u w)-t\right)-\sum_{\substack{w v \in E, w \neq u \\
v_{2}(v w)<t}}\left(v_{2}(w v)-t\right) \\
& =d_{v(G)}(u v)-\sum_{\substack{u w \in E, w \neq v \\
c_{2}<t}}\left(c_{2}-t\right)-\sum_{\substack{w v \in E, w \neq u \\
c_{2}<t}}\left(c_{2}-t\right) \\
& d_{v(G)}{ }^{(t)}(u v)=d_{v(G)}(u v) \text {, Hence } d_{G}{ }^{(t)}(u v)=d_{G}(u v) \text {. Similarly, when } c_{1} \geq t, \\
& d_{\mu(G)}{ }^{(t)}(u v)=d_{\mu(G)}(u v)-\sum_{\substack{u w \in E, w \neq v \\
\mu_{2}(u w)>t}}\left(\mu_{2}(u w)-t\right)-\sum_{\substack{w v \in E, w \neq u \\
\mu_{2}(v w)>t}}\left(\mu_{2}(w v)-t\right) \\
& =d_{\mu(G)}(u v)-\sum_{\substack{u w \in E, w \neq v \\
c_{1}>t}}\left(c_{1}-t\right)-\sum_{\substack{w v \in E, w \neq u \\
c_{1}>t}}\left(c_{1}-t\right) \\
& =d_{\mu(G)}(u v)-\left(c_{1}-t\right)\left(d_{G^{*}}(u)-1\right)-\left(c_{1}-t\right)\left(d_{G^{*}}(v)-1\right) \\
& =d_{\mu(G)}(u v)-\left(c_{1}-t\right)\left(d_{G^{*}}(u)+d_{G^{*}}(v)+2\right)=d_{\mu(G)}(u v)-\left(c_{1}-t\right) d_{G^{*}}(u v) .
\end{aligned}
$$

When, $c_{2} \leq t$,

$$
\begin{aligned}
& d_{v(G)}(t)(u v)=d_{v(G)}(u v)-\sum_{\substack{u w \in E, w \neq v \\
v_{2}(u w)<t}}\left(v_{2}(u w)-t\right)-\sum_{\substack{w v \in E, w \neq u \\
v_{2}(v w)<t}}\left(v_{2}(w v)-t\right) \\
& =d_{v(G)}(u v)-\sum_{\substack{u w \in E, w \neq v \\
c_{2}<t}}\left(c_{2}-t\right)-\sum_{\substack{w v \in E, w \neq u \\
c_{2}<t}}\left(c_{2}-t\right) \\
& =d_{v(G)}(u v)-\left(c_{2}-t\right)\left(d_{G^{*}}(u)-1\right)-\left(c_{2}-t\right)\left(d_{G^{*}}(v)-1\right) \\
& =d_{v(G)}(u v)-\left(c_{2}-t\right)\left(d_{G^{*}}(u)+d_{G^{*}}(v)-2\right)=d_{v(G)}(u v)-\left(c_{2}-t\right) d_{G^{*}}(u v)
\end{aligned}
$$

Hence, $d_{G}{ }^{(t)}(u v)=d_{G}(u v)-(c-t) d_{G^{*}}(u v)$. 


\section{Edge regular property of truncations of intuitionistic fuzzy graph}

Remark 4.1: Let $G:(V, E)$ is an edge regular intuitionistic fuzzy graph, then $G_{(\mathrm{t})}:\left(\mu_{(t)}, v_{(t)}\right)$ and $G^{(t)}:\left(\mu^{(t)}, v^{(t)}\right)$ need not be edge regular intuitionistic fuzzy graphs. For example in figure $4.1 G:(\mu, v)$ is $(1.4,1.2)$ edge regular intuitionistic fuzzy graph, but $G_{(\mathrm{t})}:\left(\mu_{(t)}, v_{(t)}\right)$ and $G^{(t)}:\left(\mu^{(t)}, v^{(t)}\right), t=(.2, .6)$ are not edge regular intuitionistic fuzzy graphs.
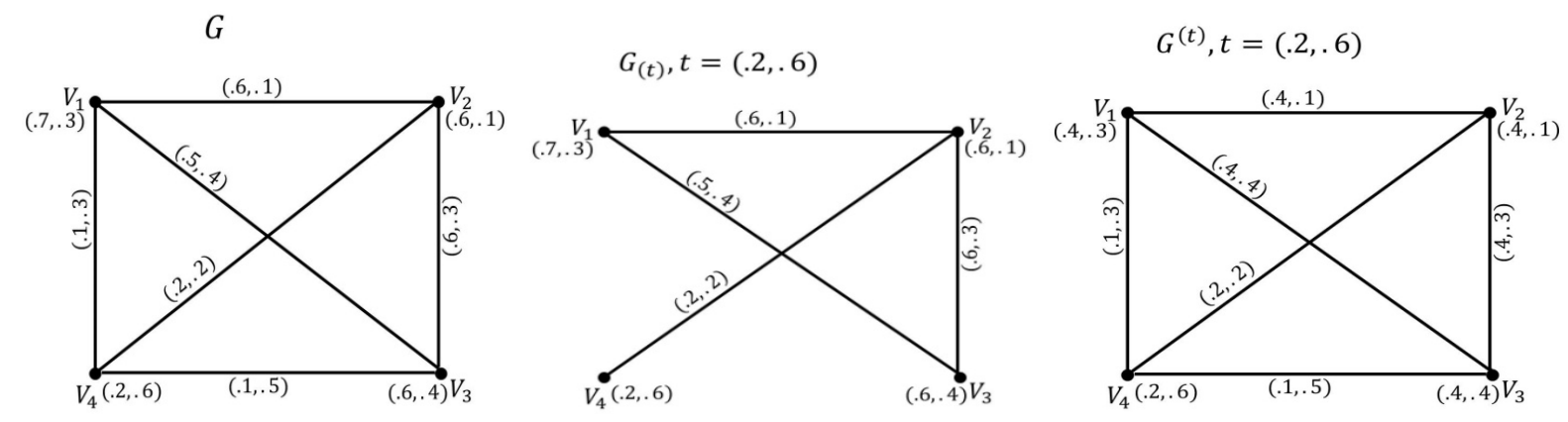

Figure 4.1

Remark 4.2: If $G_{(\mathrm{t})}:\left(\mu_{(t)}, v_{(t)}\right)$ and $G^{(t)}:\left(\mu^{(t)}, v^{(t)}\right)$ are edge regular intuitionistic fuzzy graphs, then $G:(\mu, v)$ need not be edge regular intuitionistic fuzzy graph. For example in Figure 4.2 $G_{(t)}:\left(\mu_{(t)}, v_{(t)}\right), t=(.5, .3)$ is $(.6, .3)$ edge regular and $G^{(t)}:\left(\mu^{(t)}, v^{(t)}\right), t=(.4, .2)$ is $(.8, .4)$ edge regular, but Let $G:(\mu, v)$ is not an edge regular.

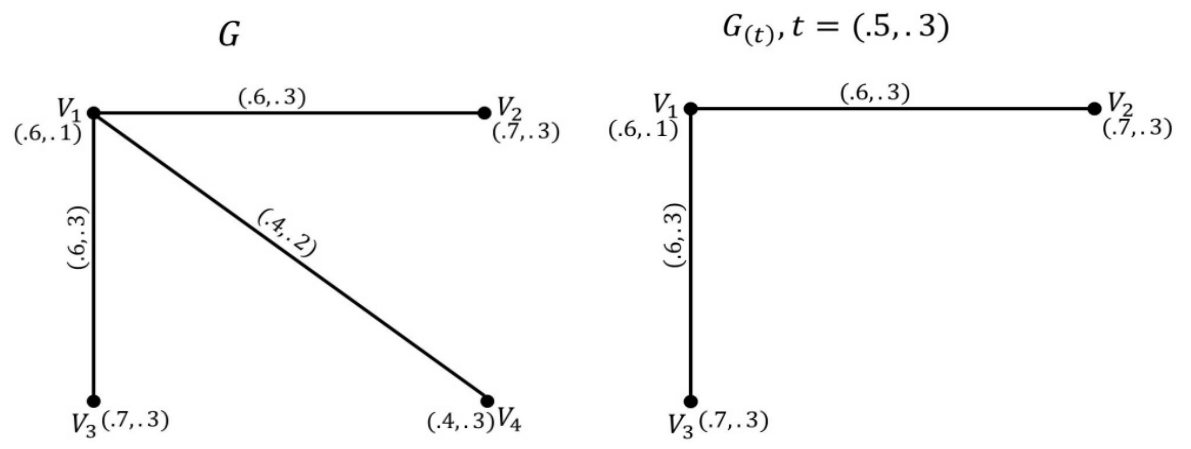

$G^{(t)}, t=(.4, .2)$

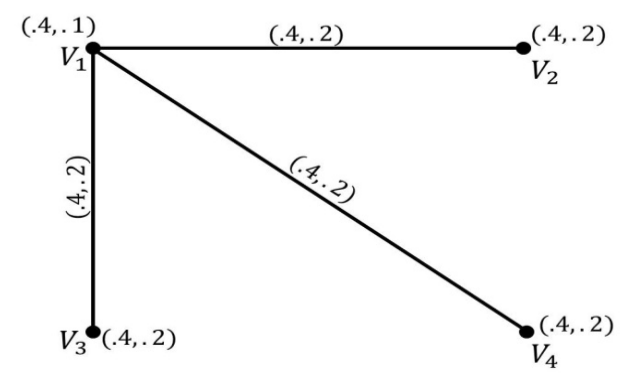

Figure 4.2

In the following Theorems, we obtain some conditions under which $G_{(t)}$ and $G^{(t)}$ are edge regular. 
Theorem 4.3: Let $G:(V, E)$ be an intuitionistic fuzzy graph such that $\mu_{2}$ and $v_{2}$ are constant functions with constant values $c_{1}$ and $c_{2}$. For every $0<t \leq c_{1} \& t \geq c_{2}, G_{(\mathrm{t})}$ is edge regular if and only if $G$ is edge regular.

Proof: If $0<t \leq c_{1} \& t \geq c_{2}$ then $V_{(\mathrm{t})}=V$, because $\mu_{1}(v) \geq t, v_{1}(v) \leq t, \forall v \in V$ and $E_{(\mathrm{t})}=E$, because $\mu_{2}(e) \geq t, v_{2}(e) \leq t, \forall e \in E$. From Theorem 3.3,

$d_{\mu(G)}(e)=d_{\mu(G)_{(t)}}(e)$ and $d_{v(G)}(e)=d_{v(G)_{(t)}}(e), \forall e \in E$

$\Rightarrow d_{G}(e)=\left(d_{\mu(G)}(e), d_{v(G)}(e)\right)=\left(d_{\mu(G)_{(t)}}(e), d_{v(G)_{(t)}}(e)\right)=d_{G_{(t)}}(e), \forall e \in E$

Hence $G$ is edge regular if and only if $G_{(\mathrm{t})}$ is edge regular.

Remark 4.4: If $c_{1}<t, c_{2}>t$, then $G_{(\mathrm{t})}$ is an empty graph.

Theorem 4.5: Let $G:(V, E)$ be an intuitionistic fuzzy graph such that $\mu_{2}$ and $v_{2}$ are constant functions. Then $G:(\mu, v)$ is an edge regular intuitionistic fuzzy graph if and only if $G^{(t)}$ is an edge regular intuitionistic fuzzy graph, where $0<t \leq 1$.

Proof: Assume that $G:(V, E)$ is an $\left(m_{1}, m_{2}\right)$ - edge regular intuitionistic fuzzy graph. Since $\mu_{2}, v_{2}$ are constant functions, by Theorem (2.9), $G^{*}:(V, E)$ is an edge regular graph. Let $G^{*}$ be $k$-edge regular. By Theorem 3.4, When $t>c_{1} \& t<c_{2}, d_{G}(t)(u v)=d_{G}(u v)=\left(m_{1}, m_{2}\right)$, $\forall u v \in E^{(t)}$. Therefore $G^{(t)}$ is $\left(m_{1}, m_{2}\right)-$ edge regular. When $t \leq c_{1} \& t \geq c_{2}, d_{G^{(t)}}(u v)=$ $\left(m_{1}, m_{2}\right)-(c-t) k \forall u v \in E^{(t)}$. Therefore $G^{(t)}$ is $\left(\left(m_{1}, m_{2}\right)-(c-t) k\right)$ - edge regular.

Conversely, assume that $G^{(t)}$ is an edge regular for every $0<t \leq 1$. When $t>c_{1}$ \& $t<c_{2}$, by Theorem $3.4 d_{G}(u v)=d_{G}{ }^{(t)}(u v), \forall u v \in E^{(t)} . \Rightarrow G$ is also edge regular. Let $t \leq$ $c_{1} \& t \geq c_{2}$.

Then $\left(\mu_{2}{ }^{(t)}, v_{2}{ }^{(t)}\right)$ is a constant function of constant value $t$. Therefore by Theorem (2.9), $G^{(t) *}$ is edge regular. Since underlying crisp graphs of $G$ and $G^{(t)}$ are same, $G^{*}$ is edge regular. From Theorem $3.4 d_{G}(u v)=d_{G}{ }^{(t)}(u v)+(c-t) d_{G^{*}}(u v) \forall u v \in E$. Hence $G$ is an edge regular intuitionistic fuzzy graph.

Theorem 4.6: Let $G:(V, E)$ be an intuitionistic fuzzy graph on an odd cycle $G^{*}$. Then $G$ is edge regular if and only if $\mu_{2}$ and $\nu_{2}$ are constant functions.

Proof: Let $G$ be a $\left(k_{1}, k_{2}\right)$ - edge regular intuitionistic fuzzy graph on an odd cycle $v_{1} v_{2}, \ldots, v_{n} v_{1}$. Let $\mu_{2}\left(v_{1} v_{2}\right)=s_{1}$ and $v_{2}\left(v_{1} v_{2}\right)=s_{2}$.

Here, $d_{\mu}\left(v_{2} v_{3}\right)=\mu_{2}\left(v_{1} v_{2}\right)+\mu_{2}\left(v_{3} v_{4}\right)=k_{1} \Rightarrow s_{1}+\mu_{2}\left(v_{3} v_{4}\right)=k_{1} \Rightarrow \mu_{2}\left(v_{3} v_{4}\right)=k_{1}-s_{1}$ $d_{v}\left(v_{2} v_{3}\right)=v_{2}\left(v_{1} v_{2}\right)+v_{2}\left(v_{3} v_{4}\right)=k_{2} \Rightarrow s_{2}+v_{2}\left(v_{3} v_{4}\right)=k_{2} \Rightarrow v_{2}\left(v_{3} v_{4}\right)=k_{2}-s_{2}$

Similarly, $\mu_{2}\left(v_{5} v_{6}\right)=s_{1}, \mu_{2}\left(v_{7} v_{8}\right)=k_{1}-s_{1}$ and so on, $v_{2}\left(v_{5} v_{6}\right)=s_{2}, v_{2}\left(v_{7} v_{8}\right)=k_{2}-s_{2}$ and so on. Proceeding this way,

and

$$
\mu_{2}\left(v_{n} v_{1}\right)=\left\{\begin{array}{c}
s_{1} \text { if } n-1 \equiv 0(\bmod 4) \\
k_{1}-s_{1} \text { if } n-1 \neq 0(\bmod 4)
\end{array}\right.
$$

$$
v_{2}\left(v_{n} v_{1}\right)=\left\{\begin{array}{c}
s_{2} \text { if } n-1 \equiv 0(\bmod 4) \\
k_{2}-s_{2} \text { if } n-1 \neq 0(\bmod 4)
\end{array}\right.
$$

Case $(i): \mu_{2}\left(v_{n} v_{1}\right)=s_{1}$ and $v_{2}\left(v_{n} v_{1}\right)=s_{2}$. Therefore $\mu_{2}\left(v_{2} v_{3}\right)=k_{1}-s_{1}, \mu_{2}\left(v_{4} v_{5}\right)=s_{1}$, $\mu_{2}\left(v_{6} v_{7}\right)=k_{1}-s_{1}$ and so on. Since $n-1 \equiv 0(\bmod 4), \mu_{2}\left(v_{n-1} v_{n}\right)=s_{1}$.

$$
d_{\mu}\left(v_{n} v_{1}\right)=k \Rightarrow \mu_{2}\left(v_{n} v_{1}\right)+\mu_{2}\left(v_{1} v_{2}\right)=k_{1} \Rightarrow s_{1}+s_{1}=k_{1} \Rightarrow s_{1}=\frac{k_{1}}{2} \text {. }
$$


Therefore $k_{1}-s_{1}=k_{1}-\frac{k_{1}}{2}=\frac{k_{1}}{2} \cdot \mu_{2}\left(v_{i} v_{i+1}\right)=\frac{k_{1}}{2}, \forall i=1,2,3, \ldots n$, where $v_{n+1}=v_{1}$.

Similarly we get $s_{2}=\frac{k_{2}}{2} \cdot v_{2}\left(v_{i} v_{i+1}\right)=\frac{k_{2}}{2}, \forall i=1,2,3, \ldots n$, Where $v_{n+1}=v_{1}$.

Case (ii): $\mu_{2}\left(v_{n} v_{1}\right)=k_{1}-s_{1}$ and $v_{2}\left(v_{n} v_{1}\right)=k_{2}-s_{2}$.

Proceeding as above, $\mu_{2}\left(v_{2} v_{3}\right)=s_{1}, \mu_{2}\left(v_{4} v_{5}\right)=k_{1}-s_{1}, \mu_{2}\left(v_{6} v_{7}\right)=s_{1}$ and so on, $v_{2}\left(v_{2} v_{3}\right)=s_{2}, v_{2}\left(v_{4} v_{5}\right)=k_{2}-s_{2}, v_{2}\left(v_{6} v_{7}\right)=s_{2}$ and so on.

Since $n-1 \not \equiv 0(\bmod 4), \mu_{2}\left(v_{n-1} v_{n}\right)=s_{1}$ and $v_{2}\left(v_{n-1} v_{n}\right)=s_{2}$

Now proceeding as above, $d_{\mu}\left(v_{n} v_{1}\right)=k_{1} \Rightarrow \mu_{2}\left(v_{n-1} v_{n}\right)+\mu_{2}\left(v_{1} v_{2}\right)=k_{1} \Rightarrow s_{1}+s_{1}=k_{1}$

$\Rightarrow s_{1}=\frac{k_{1}}{2}, \mu_{2}\left(v_{i} v_{i+1}\right)=\frac{k_{1}}{2}, \forall i=1,2, \ldots n$, where $v_{n+1}=v_{1}$. Similarly we get $s_{2}=\frac{k_{2}}{2}$,

$v_{2}\left(v_{i} v_{i+1}\right)=\frac{k_{2}}{2}, \forall i=1,2, \ldots n$, where $v_{n+1}=v_{1}$. Hence in the both cases, $\mu_{2}$ and $v_{2}$ are constant functions. Conversely, assume that $\mu_{2}$ and $v_{2}$ are constant functions with constant values $c_{1}$ and $c_{2}$ respectively. Then $d_{\mu}\left(v_{i} v_{i+1}\right)=2 c_{1}$ and $d_{v}\left(v_{i} v_{i+1}\right)=2 c_{2}$. Therefore $G$ is a $\left(2 c_{1}, 2 c_{2}\right)$ - edge regular.

Theorem 4.7: Let $G:(V, E)$ be an intuitionistic fuzzy graph on an even cycle $G^{*}$ with $n$ vertices and let $n \not \equiv 0(\bmod 4)$. Then $G$ is an edge regular intuitionistic fuzzy graph if and only if $\mu_{2}$ and $v_{2}$ are constant functions.

Proof: Let $G$ be an intuitionistic fuzzy graph on an even cycle $v_{1} v_{2}, \ldots, v_{n} v_{1}$, where $n \not \equiv$ $0(\bmod 4)$. If $\mu_{2} \& v_{2}$ are constant functions with constant values $c_{1}$ and $c_{2}$ respectively.

$d_{G}\left(v_{i} v_{i+1}\right)=\left(d_{\mu}\left(v_{i} v_{i+1}\right), d_{v}\left(v_{i} v_{i+1}\right)\right)=\left(2 c_{1}, 2 c_{2}\right)$.

Therefore $G$ is a $\left(2 c_{1}, 2 c_{2}\right)$ - edge regular intuitionistic fuzzy graph.

Conversely, let $G$ is a $\left(k_{1}, k_{2}\right)$ - edge regular intuitionistic fuzzy graph. Since $n$ is even and $n \not \equiv 0(\bmod 4)$, we have $n-2 \equiv 0(\bmod 4)$. Therefore the number of edges that lie alternatively from $v_{1} v_{2}$ is $\frac{n}{2}-1$ (an even number).Let $\mu_{2}\left(v_{1} v_{2}\right)=s_{1}$ and $v_{2}\left(v_{1} v_{2}\right)=s_{2}$. Then proceeding as above $\mu_{2}\left(v_{3} v_{4}\right)=k_{1}-s_{1}$ and $v_{2}\left(v_{3} v_{4}\right)=k_{2}-s_{2}, \mu_{2}\left(v_{5} v_{6}\right)=s_{1}$ and $v_{2}\left(v_{5} v_{6}\right)=s_{2}, \ldots, \mu_{2}\left(v_{n-3} v_{n-2}\right)=k_{1}-s_{1}$ and $v_{2}\left(v_{n-3} v_{n-2}\right)=k_{2}-s_{2}, \mu_{2}\left(v_{n-1} v_{n}\right)=s_{1}$ and $v_{2}\left(v_{n-1} v_{n}\right)=s_{2}$.

$$
d_{\mu}\left(v_{n} v_{1}\right)=\mu_{2}\left(v_{n-1} v_{n}\right)+\mu_{2}\left(v_{1} v_{2}\right)=k_{1} \Rightarrow s_{1}+s_{1}=k_{1} \Rightarrow s_{1}=\frac{k_{1}}{2} \text {. }
$$

Therefore $k_{1}-s_{1}=\frac{k_{1}}{2}$, Similarly $s_{2}=\frac{k_{2}}{2}$ and $k_{2}-s_{2}=\frac{k_{2}}{2}$.

$\mu_{2}\left(v_{1} v_{2}\right)=\mu_{2}\left(v_{3} v_{4}\right)=\cdots=\mu_{2}\left(v_{n-1} v_{n}\right)=\frac{k_{1}}{2}, v_{2}\left(v_{1} v_{2}\right)=v_{2}\left(v_{3} v_{4}\right)=\cdots=v_{2}\left(v_{n-1} v_{n}\right)=$ $\frac{k_{2}}{2}$. Similarly if $\mu_{2}\left(v_{2} v_{3}\right)=r_{1}$ and $v_{2}\left(v_{2} v_{3}\right)=r_{2}$ then proceeding as above, $\mu_{2}\left(v_{n} v_{1}\right)=r_{1}$ and $v_{2}\left(v_{n} v_{1}\right)=r_{2} \cdot d_{\mu}\left(v_{1} v_{2}\right)=k_{1}$ and $d_{v}\left(v_{1} v_{2}\right)=k_{2}, \Rightarrow r_{1}=\frac{k_{1}}{2}$ and $r_{2}=\frac{k_{2}}{2}$.

Therefore $\mu_{2}$ and $v_{2}$ are constant functions.

Theorem 4.8: Let $G:(V, E)$ be an intuitionistic fuzzy graph on an even cycle $G^{*}$ with $n$ vertices and let $n \equiv 0(\bmod 4)$. Then $G$ is a $\left(k_{1}, k_{2}\right)$ - edge regular intuitionistic fuzzy graph if and only if $\mu_{2}$ and $v_{2}$ assumes exactly eight values $r_{i}, s_{i}, t_{i}$ and $l_{i}, i=1,2$ such that consecutive adjacent edges receives these values in cyclic order with $r_{i}+t_{i}=k_{i}$ and $s_{i}+l_{i}=k_{i}, i=1,2$.

Proof: Let $G$ be a $\left(k_{1}, k_{2}\right)$-edge regular intuitionistic fuzzy graph on an even cycle $v_{1} v_{2}, \ldots, v_{n} v_{1}$, where $n \equiv 0(\bmod 4)$. Let $\mu_{2}\left(v_{1} v_{2}\right)=r_{1}$ and $v_{2}\left(v_{1} v_{2}\right)=r_{2}$.

Since $n \equiv 0(\bmod 4), n-2$ is an even and $n-2 \not \equiv 0(\bmod 4)$. 
$\Rightarrow \frac{n}{2}-1$ is an odd number. Therefore the number of edges that lie alternatively from $v_{1} v_{2}$ is $\frac{n}{2}-1$ (an odd number). Now $\mu_{2}\left(v_{1} v_{2}\right)=r_{1}$ and $\mu_{2}\left(v_{2} v_{3}\right)=k_{1} \Rightarrow \mu_{2}\left(v_{3} v_{4}\right)=k_{1}-r_{1}$.

Similarly, $\mu_{2}\left(v_{5} v_{6}\right)=r_{1}, \ldots, \mu_{2}\left(v_{n-1} v_{n}\right)=k_{1}-r_{1}$. If $\mu_{2}\left(v_{2} v_{3}\right)=s_{1}$, then proceeding as above $\mu_{2}\left(v_{4} v_{5}\right)=k_{1}-s_{1}, \mu_{2}\left(v_{6} v_{7}\right)=s_{1}, \ldots, \mu_{2}\left(v_{n} v_{1}\right)=k_{1}-s_{1}$.

Thus the consecutive adjacent edges of the cycle receives the four membership values $r_{1}, s_{1}, k_{1}-r_{1}$ and $k_{1}-s_{1}$ in cyclic order. Similarly the consecutive adjacent edges of the cycle receives the four non membership values $r_{2}, s_{2}, k_{2}-r_{2}$ and $k_{2}-s_{2}$ in cyclic order. Conversely, assume that $\mu_{2}$ and $v_{2}$ exactly takes eight values respectively as $r_{i}, s_{i}, t_{i}$ and $l_{i}, i=1,2$ such that consecutive adjacent edges receives these values in cyclic order with $r_{i}+t_{i}=k_{i}$ and $s_{i}+l_{i}=$ $k_{i}, i=1,2 . \quad d(e)=\left(r_{1}+t_{1}, r_{2}+t_{2}\right)($ or $)\left(s_{1}+l_{1}, s_{2}+l_{2}\right)=\left(k_{1}, k_{2}\right)($ or $)\left(k_{1}, k_{2}\right) \Rightarrow d(e)=$ $\left(k_{1}, k_{2}\right) \forall e \in E$.

Therefore $G$ is a $\left(k_{1}, k_{2}\right)$ - edge regular intuitionistic fuzzy graph.

Theorem 4.9: Let $G:(V, E)$ be an intuitionistic fuzzy graph on a cycle $G^{*}$ with $n \not \equiv 0(\bmod 4)$, where $|V|=n$. Then $G:(\mu, v)$ is an edge regular intuitionistic fuzzy graph if and only if $G^{(t)}$ is an edge regular intuitionistic fuzzy graph, where $0<t \leq 1$.

Proof: Given $G$ is an intuitionistic fuzzy graph on a cycle $G^{*}$ with $n \not \equiv 0(\bmod 4)$. Then by Theorem 4.6 and 4.7, we have $\mu_{2}$ and $\nu_{2}$ are constant functions then $G$ is an edge regular intuitionistic fuzzy graph if and only if $G^{(t)}$ is an edge regular intuitionistic fuzzy graph, where $0<t \leq 1$.

Definition 4.10: The adjacency sequence of a vertex $v$ in an intuitionistic fuzzy graph $G$ is defined as a sequence of both membership and non membership values of edges incident at $v$ arranged in increasing order. It is denoted by $a s(v)$.

Definition 4.11: The adjacency sequence of an edge $e$ in an intuitionistic fuzzy graph $G$ is defined as a sequence of both membership and non membership values of edges adjacent to $e$ arranged in increasing order. It is denoted by $a s(e)$.

Theorem 4.12: If all the edges of $G$ have the same adjacency sequence, then all the edges of $G_{(t)}$ have the same adjacency sequence.

Proof: Suppose that all the edges of $G$ have the same adjacency sequence, say $\left(k_{1}, k_{2}, \ldots, k_{n}\right)$ in membership and $\left(s_{1}, s_{2}, \ldots, s_{n}\right)$ in non membership. If $t>k_{n}$ and $t<s_{n}$, then there is no edge in $G_{(t)}$. If $t \leq k_{1}$ and $t \geq s_{1}$, then adjacency sequence of $e$ is $\left(k_{1}, k_{2}, \ldots, k_{n}\right)$ in membership and $\left(s_{1}, s_{2}, \ldots, s_{n}\right)$ in non membership for each $e \in E_{(t)}$.

If $k_{i-1}<t \leq k_{i}$ and $s_{i-1}>t \geq s_{i}$, then the adjacency sequence of $e$ is $\left(k_{i}, k_{i+1}, \ldots, k_{n}\right)$ in membership and $\left(s_{i}, s_{i+1}, \ldots, s_{n}\right)$ in non-membership for each $e \in E_{(t)}$.

Remark 4.13: Converse of Theorem 4.13 need not be true. For example all the edges in $G_{(t)}, t=(.5, .3)$ have the same adjacency sequence $((.6, .2),(.7, .3))$. But in $G$,

$$
\operatorname{as}(p)=((.4, .1),(.6, .2),(.7, .3)) \neq((.6, .2),(.6, .2),(.7, .3),(.7, .3))=\operatorname{as}(t)
$$


$G$

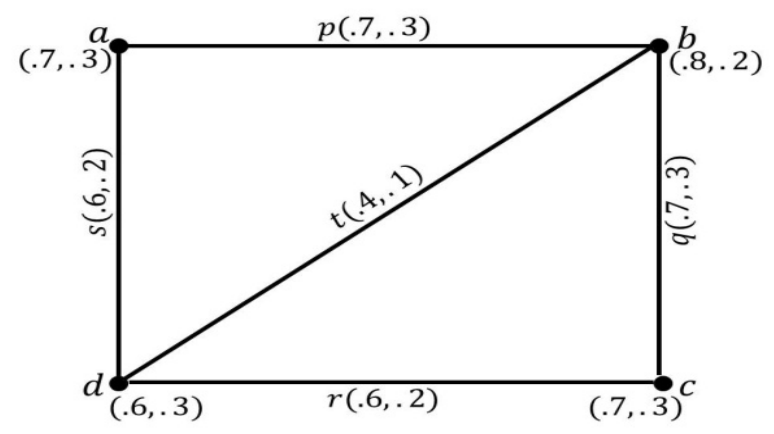

$G_{(t)}, t=(.5, .3)$

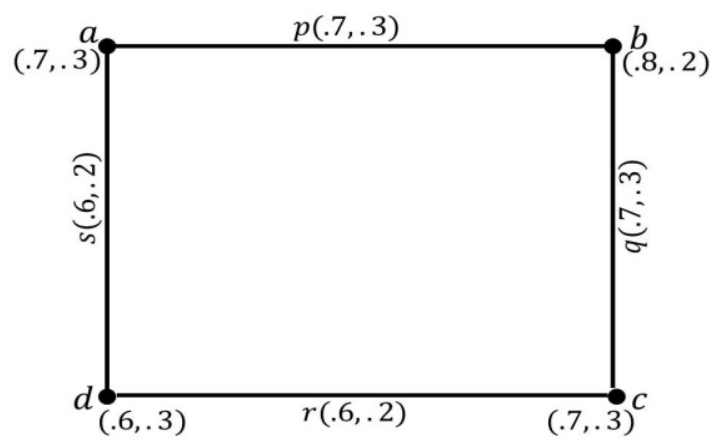

Figure 4.3

Theorem 4.14: If all the edges of $G$ have the same adjacency sequence, then all the edges of $G^{(t)}$ have the same adjacency sequence.

Proof: Suppose that all the edges of $G$ have the same adjacency sequence, say $\left(k_{1}, k_{2}, \ldots, k_{n}\right)$ and $\left(s_{1}, s_{2}, \ldots, s_{n}\right)$ in membership and non membership respectively in $G$. If $t>k_{n}$ and $t<s_{n}$, then $\left(k_{1}, k_{2}, \ldots, k_{n}\right)$ and $\left(s_{1}, s_{2}, \ldots, s_{n}\right)$ are adjacency sequence in membership and non membership for each $e \in E^{(t)}$. If $t \leq k_{1}$ and $t \geq s_{1}$, then $(t, t, \ldots, t)$ and $(t, t, \ldots, t)$ are adjacency sequence in membership and non membership for each $e \in E^{(t)}$.

If $k_{i-1}<t \leq k_{i}$ and $s_{i-1}>t \geq s_{i}$, then the adjacency sequence in membership and non-membership is $\left(k_{1}, k_{2}, \ldots, k_{i-1}, t, t, \ldots, t\right)$ and $\left(s_{1}, s_{2}, \ldots, s_{i-1}, t, t, \ldots, t\right)$ respectively, $\forall e \in E^{(t)}$.

Remark 4.15: Converse of Theorem 4.15 need not be true. For example all the edges in $G^{(t)}, t=$ $(.4, .2)$ have the same adjacency sequence $((.2, .1),(.4, .2),(.4, .2))$. But in $G$, $\operatorname{as}(p)=((.2, .1),(.5, .2),(.7, .3)) \neq((.2, .1),(.6, .25),(.7, .3))=\operatorname{as}(q)$

\section{$G$}

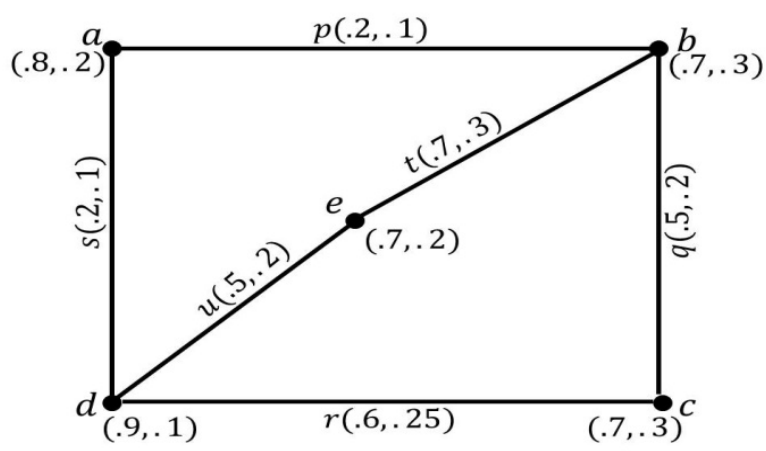

$G^{(t)}, t=(0.4,0.2)$

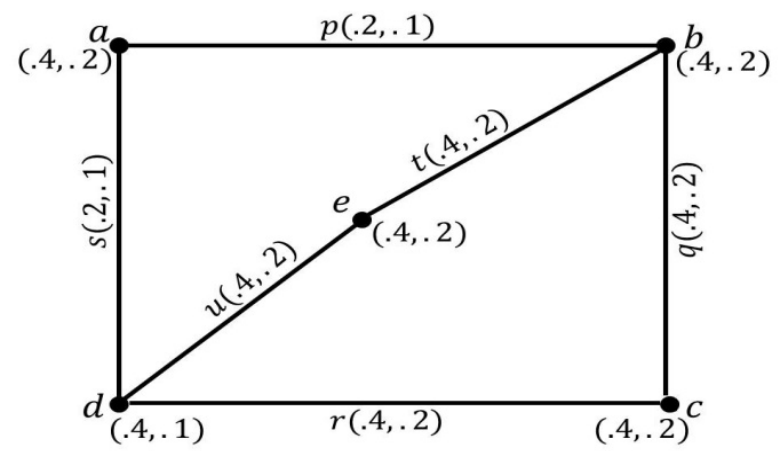

Figure 4.4

Theorem 4.16: If all the edges of $G$ have the same adjacency sequence, then $G_{(t)}$ and $G^{(t)}$ are edge regular intuitionistic fuzzy graphs. 
Proof: When all the edges of $G$ have the same adjacency sequence, the same holds for $G_{(t)}$ and $G^{(t)}$ also. Since the sum of all the elements of the adjacency sequence of an edge is its degree $d_{G}(e)=\left(d_{\mu}(e), d_{v}(e)\right), G_{(t)}$ and $G^{(t)}$ are edge regular intuitionistic fuzzy graphs.

\section{References}

[1] Atanassov, K. (1999) Intuitionistic Fuzzy Sets: Theory and Applications, Springer-Physica Verlag, Heidelberg.

[2] Guo-jun, W. \& He, Y.-Y, (2000) Intuitionistic Fuzzy Sets and L-Fuzzy Sets, Fuzzy Sets and Systems, 110(2), 271-274.

[3] Karunambigai, M. G., Palanivel, K., \& Sivasankar, S., (2015) Edge regular intuitionistic fuzzy graph, Advances in Fuzzy Sets and Systems, 20(1), 25-46.

[4] Karunambigai, M. G., Sivasankar, S. \& Palanivel, K. (2015) Some properties of regular intuitionistic fuzzy graph, International Journal of Mathematics and Computation, 26(4), $138-146$.

[5] Muhammad, A., \& Parvathi, R. (2012) Properties of intuitionistic fuzzy line graphs, Notes on Intuitionistic Fuzzy Sets, 18(3), 52-60.

[6] Muhammad, A. \& Alshehri, N. O. (2014) The Intuitionistic Fuzzy Cycle Intuitionistic Fuzzy Trees, The Scientific World Journal, Volume 2014, Article ID 305836, 11 pages.

[7] Nagoor Gani, A., \& Sheik Mujibur Rahman, H. (2017) Truncations on Special Intuitionistic Fuzzy Graphs, Accepted in International Journal of Pure and Applied Mathematics, Bulgaria.

[8] Parvathi, R., Karunambigai, M. G., \& Atanassov, K. (2009) Operations on intuitionistic fuzzy graphs, Proc. of IEEE International Conference on Fuzzy Systems '2009, 1396-1401.

[9] Radha, K., \& Kumaravel, N. (2016) The Edge Degree and the Edge Regular Properties of Truncations of Fuzzy Graphs, Bulletin of Mathematics and Statistics Research, 4(3), $7-16$. 